\title{
A homopolar disc dynamo experiment with liquid metal contacts
}

\author{
R. A. Avalos-Zúñiga ${ }^{1}, J$. Priede ${ }^{2}$, C. E. Bello-Morales ${ }^{1}$ \\ 1 CICATA-Qro, Instituto Politécnico Nacional, Cerro Blanco 141, Colinas del \\ Cimatario, 76090, Querétaro, Mexico \\ ${ }^{2}$ Applied Mathematics Research Centre, Coventry University, Coventry, CV1 5FB, UK
}

\begin{abstract}
We present experimental results of a homopolar disc dynamo constructed at CICATAQuerétaro in Mexico. The device consists of a flat, multi-arm spiral coil which is placed above a fast-spinning metal disc and connected to the latter by sliding liquid-metal electrical contacts. Theoretically, self-excitation of the magnetic field is expected at the critical magnetic Reynolds number $R m \approx 45$, which corresponds to a critical rotation rate of about $10 \mathrm{~Hz}$. We measured the magnetic field above the disc and the voltage drop on the coil for the rotation rate up to $14 \mathrm{~Hz}$, at which the liquid metal started to leak from the outer sliding contact. Instead of the steady magnetic field predicted by the theory we detected a strongly fluctuating magnetic field with a strength comparable to that of Earth's magnetic field which was accompanied by similar voltage fluctuations in the coil. These fluctuations seem to be caused by the intermittent electrical contact through the liquid metal. The experimental results suggest that the dynamo with the actual electrical resistance of liquid metal contacts could be excited at the rotation rate of around $21 \mathrm{~Hz}$ provided that the leakage of liquid metal is prevented.
\end{abstract}

Introduction The homopolar disc dynamo is one of the simplest models of the self-excitation of magnetic field by moving conductors which is often used to illustrate the dynamo effect [1, 2]. In its simplest form originally considered by Bullard [3], the dynamo consists of a solid metal disc which rotates about its axis and a wire twisted around it which is connected through sliding contacts to the rim and to the axis of the disc. For a sufficiently high rotation rate, the voltage induced by the rotation of the disc in a magnetic field generated by an initial current perturbation can exceed the voltage drop due to the ohmic resistance. At this point, the initial perturbation starts to grow exponentially leading to the selfexcitation of current and the associated magnetic field. Despite its simplicity, no successful experimental implementation of the disc dynamo is known so far. The problem appears to be the sliding electrical contacts which are required to convey the current between the rim and the axis of the rotating disc. The electrical resistance of the sliding contacts, usually made of solid graphite brushes, is typically by several orders of magnitude higher than that of the rest of the setup [4. This results in unrealistically high rotation rates which are required for dynamo to operate. To overcome this problem Priede et al. [5] proposed a theoretical design of homopolar disc dynamo which uses liquid-metal sliding electrical contacts similar to those employed in homopolar motors and generators [6, 7]. The design consists of a flat multi-arm spiral coil placed above a fast-spinning copper disc and connected to the latter by sliding liquid-metal electrical contacts. The theoretical results obtained in [5] show a minimum magnetic Reynolds number of 40, at which the dynamo is self-excited. This can be achieved by using a copper disc of $60 \mathrm{~cm}$ in diameter which rotates with the frequency of $10 \mathrm{~Hz}$. In this paper, we describe a laboratory model such a disc dynamo and report the first results of its operation. 

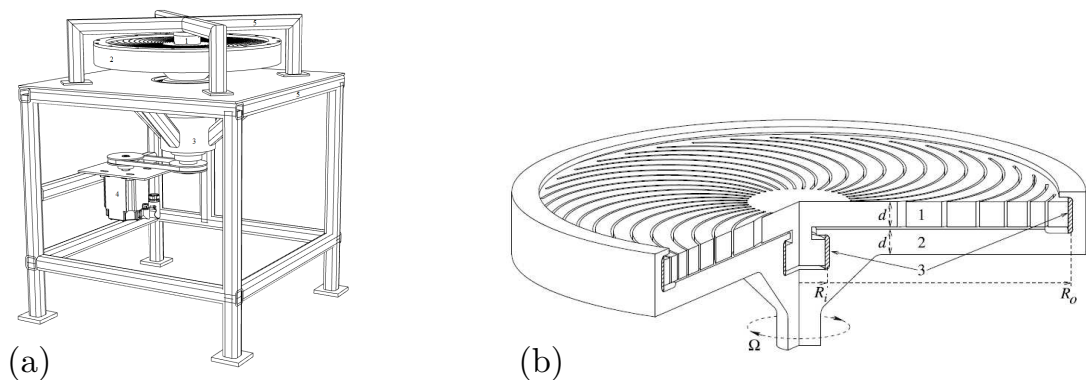

(b)

Figure 1: (a) Schematic view of the dynamo facility showing stationary coil (1), fast-spinning disc (2), gearing system (3), AC motor (4), and iron frame(5); (b) cross-sectional view of the disc dynamo setup consisting of a stationary coil (1) made of a copper disc sectioned by spiral slits and a fast-spinning disc (2), which is electrically connected to the former by sliding liquid-metal contacts (hatched) (3).

1. Description of experimental setup The dynamo setup shown in Fig. 1 consists of a fast-spinning copper disc and a coil made of a stationary copper disc which is sectioned by 40 logarithmic spiral slits with a constant pitch angle $\alpha \approx 58^{\circ}$ relative to the radial direction. The rotating disc includes a central cylindrical cavity and an annular cavity at its rim. The coil disc has a cylindrical solid electrode protruding $4 \mathrm{~cm}$ out from the center of its bottom face. It is inserted $3 \mathrm{~mm}$ over the rotating disc surface forming the inner and outer annular gaps of width $\delta=3 \mathrm{~mm}$ and height $d=3 \mathrm{~cm}$, with inner and outer radii $R_{i}=4.5 \mathrm{~cm}$ and $R_{o}=30 \mathrm{~cm}$, respectively. At the bottom of the annular gaps, there is an eutectic alloy of GaInSn which is liquid at the room temperature. As the rotation rate of the disc increases the centrifugal force pushes the liquid metal through the gaps and creates sliding contacts which connect electrically the stationary coil and the rotating disc. At the top of the sliding contact, a ring cap with 40 thin vanes at its bottom face is attached to the rotating disc. This creates a centrifugal pump which acts as a dynamic seal for the liquid metal. The coil and disc are hold by iron supports which are electrically isolated from the copper discs. The spinning disc is driven by a $3 \mathrm{hp} \mathrm{AC} \mathrm{motor} \mathrm{through} \mathrm{a} \mathrm{gearing} \mathrm{system.} \mathrm{The} \mathrm{motor} \mathrm{rotation}$ rate is controlled by a variable frequency drive (VFD) from 0.5 to $20 \mathrm{~Hz}$ which correspond to $R m=2 \cdots 86$.

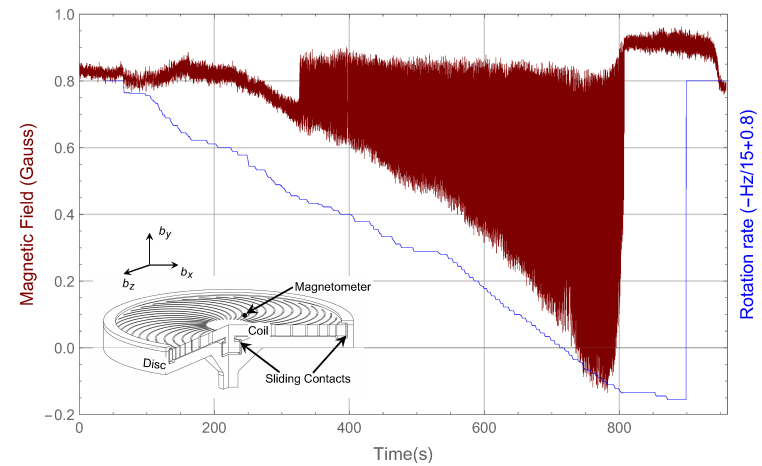

Figure 2: Rotation rate and the vertical component $\left(B_{y}\right)$ of the magnetic field versus time. 
2. Magnetic field and voltage measurements We report measurements of the temporal variation of the magnetic field and the induced voltage at the upper face of the coil for rotation rates of the disc ranging from 0.5 to $14 \mathrm{~Hz}$. The sense of rotation is opposite to the orientation of the spiral arms of the coil which corresponds to a clock-wise rotation in the setup shown in Fig. 1(b). The measurements of the induced magnetic field were performed with a three-axis Hall sensor (Low-Field METROLAB magnetometer 1176LF) placed at the upper face of the coil close to the inner radius of the spiral slits. The rate of data acquisition was 30 samples per second for a period of 14 minutes. In Figure 2 we show the temporal variation of the vertical component $\left(b_{y}\right)$ of the measured magnetic field together with the rotation rate of the disc. For a rotation frequency of $4.5 \mathrm{~Hz}$ the field starts to decrease nearly linearly with the increase of the rotation rate. Then around the rotation frequency of $5.45 \mathrm{~Hz}$ the field starts to oscillate between $0.66-0.87 \mathrm{G}$ and $0.735-0.75 \mathrm{G}$ in approximately one second (see Fig. 31). During the maximum phase, the field returns close its background value, which was measured at low rotation rates. The background magnetic field, which is important for the interpretation of experimental results, will be discussed in the next section. Such oscillations persist up to the rotation frequency of $13.5 \mathrm{~Hz}$. At this point the period of field oscillations abruptly increased to 22.5 seconds and stayed nearly the same as the disc rotation rate was continuously increased up to its highest value of $14 \mathrm{~Hz}$.

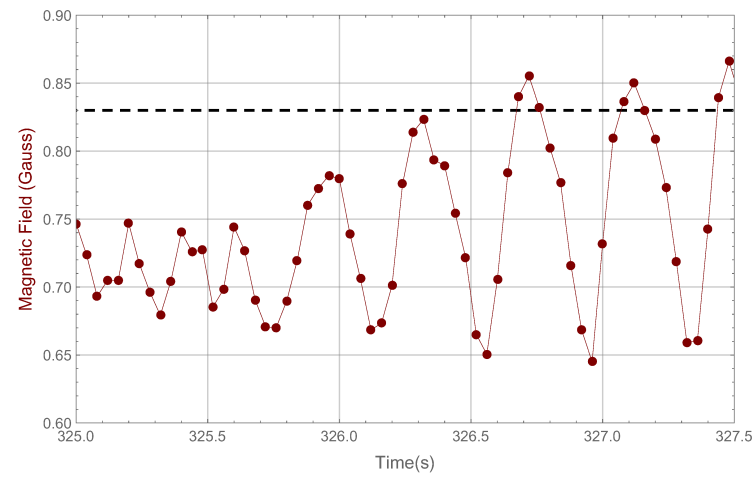

Figure 3: Temporal variation of the vertical component $\left(b_{y}\right)$ of the magnetic field for the rotation rate of $5.45 \mathrm{~Hz}$. Dashed line corresponds to the background magnetic field.

Voltage was measured at the upper face of the coil between the inner and outer radius of spiral arm (see sketch in Fig. 4) using a digital multimeter (Keithley 2100). The data was acquired with a rate of 10 samples per second for a period of 14 minutes. In Figure 4 we show the temporal variation of the induced voltage together with the rotation rate of the disc. Contrary to the magnetic field, the variation of the induced voltage was observable as soon as the disc started to rotate. At the rotation frequencies higher than $5 \mathrm{~Hz}$ the voltage was found to vary similarly to the magnetic field. 


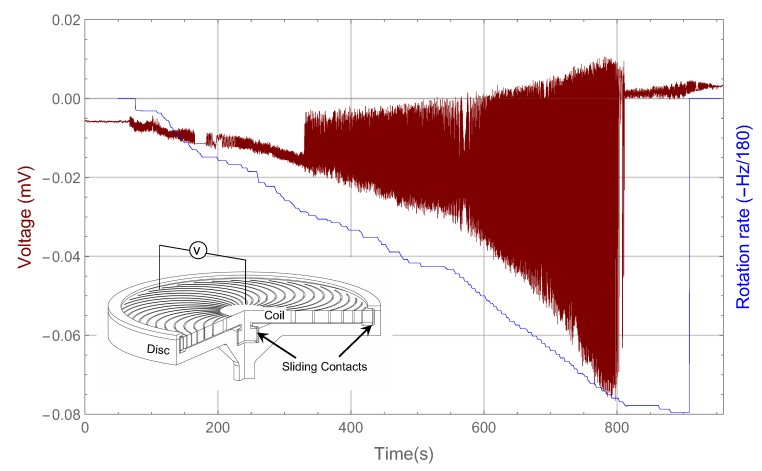

Figure 4: Rotation rate and the coil voltage versus time.

3. Interpretation of experimental data In order to interpret the experimental results presented above we need take into account that they were obtained in the presence of a rather complicated background magnetic field. First of all, there was Earth's magnetic field with a downward (negative) vertical component of strength $0.32 \mathrm{G}$. However, the Hall sensor, which was attached close to the iron frame holding the coil, measured a background magnetic field with an upward (positive) vertical component of strength $0.84 \mathrm{G}$. In the absence of more detailed measurements it seems reasonable to assume that the measured background magnetic field is mostly due to the magnetization of the iron frame and, thus, localized in its vicinity while the disc is largely subject to Earth's magnetic field. Then a subcritical amplification of Earth's magnetic field, which is opposite to that of iron frame at the sensor location, may explain the observed reduction in the measured magnetic field. It has to be noted that there is only amplification but no flux expulsion effect in our dynamo which could provide an alternative explanation. In order to test this hypothesis, let us estimate the current, voltage and magnetic field induced by the disc rotating with a subcritical (below the dynamo threshold) frequency in Earth's magnetic field. Taking into account the background magnetic field with the vertical component $B_{0}$ and the associated angular flux density $\phi_{0}=\int_{R_{i}}^{R_{o}} B_{0} r d r \approx B_{0}\left(R_{o}^{2}-R_{i}^{2}\right) / 2$, the voltage induced across the disc rotating with angular velocity $\Omega$ can be written according to the theoretical model of Priede et al. [5] as

$$
\Delta \varphi_{d}=\Omega\left(\phi_{0}+\phi_{d}\right)+\frac{I_{0} \ln (\lambda)}{2 \pi \sigma d}
$$

where

$$
\phi_{d}=\frac{\beta \mu_{0} I_{0} R_{o}}{8 \pi^{2}} \bar{\phi}(\lambda)
$$

is the magnetic flux density induced by the current $I_{0}$ flowing across the copper coil with the conductivity $\sigma=5.96 \times 10^{7} \mathrm{~S} / \mathrm{m}$ and the helicity $\beta=\tan \left(58^{\circ}\right) \approx 1.6$ of the logarithmic spiral arms; $\lambda=R_{0} / R_{i}=1 / 6$ is the radii ratio for which $\bar{\phi}(\lambda)$ the dimensionless flux $\bar{\phi}(\lambda) \approx 1.7[5] ; \mu_{0}=4 \pi \times 10^{-7} \mathrm{H} / \mathrm{m}$ is the permeability of vacuum. The current is related with the coil voltage by Ohm's law:

$$
\Delta \varphi_{c}=-I_{0} \frac{\left(1+\beta^{2}\right) \ln \lambda}{2 \pi \sigma d} .
$$

which allows us to determine $I_{0}$ and other related quantities from the measured values of $\Delta \varphi_{c}$. On the other hand, applying Ohm's law to the whole electrical 
circuit including the disc, coil and liquid-metal contacts we obtain

$$
\Delta \varphi_{d}-\Delta \varphi_{c}=I_{0} \mathcal{R},
$$

where $\mathcal{R}$ is a liquid-metal contact resistance. This relation allows us to evaluate the actual contact resistance from the measured coil voltage. The results are shown in Table 1 in terms of the dimensionless contact resistance $\overline{\mathcal{R}}=2 \pi \sigma d \mathcal{R}$, which is seen to be about an order of magnitude higher than its theoretical estimate $\overline{\mathcal{R}} \approx 1.26$ [5]. Such a high contact resistance may due to the heavy oxidation of the eutectic alloy of GaInSn which was observed in experiments. As a result, the rotation rates at which dynamo could become self-sustained are around $25 \mathrm{~Hz}$ which are about a factor of two higher than the theoretical value.

\begin{tabular}{|c|c|c|c|}
\hline$\Omega(\mathrm{Hz})$ & $\mathcal{R}$ & $R m_{c}$ & $\Omega_{c}(\mathrm{~Hz})$ \\
\hline \hline 6 & 12.3 & 97 & 22.9 \\
\hline 10 & 15.1 & 110 & 25.9 \\
\hline 14 & 15.2 & 110.5 & 26.1 \\
\hline
\end{tabular}

Table 1: The dimensionless contact resistance $\overline{\mathcal{R}}$ and the corresponding critical values $R m_{c}$ and $\Omega_{c}$ evaluated using the coil voltage measured at different rotation rates.

On the other hand, combining the equations above we can write the induced magnetic flux density as

$$
\phi_{d}=\phi_{0} \Omega /\left(\Omega_{c}-\Omega\right)
$$

where $\Omega_{c}$ is a critical rotation rate at which the dynamo becomes self-sustained. A similar expressions hold also for the induced current and voltage. Using this expression to fit the measurements in Fig. 5, we find $\Omega_{c} \approx 21 \mathrm{~Hz}$, which is consistent with the results based on the actual contact resistance shown in Table 1.
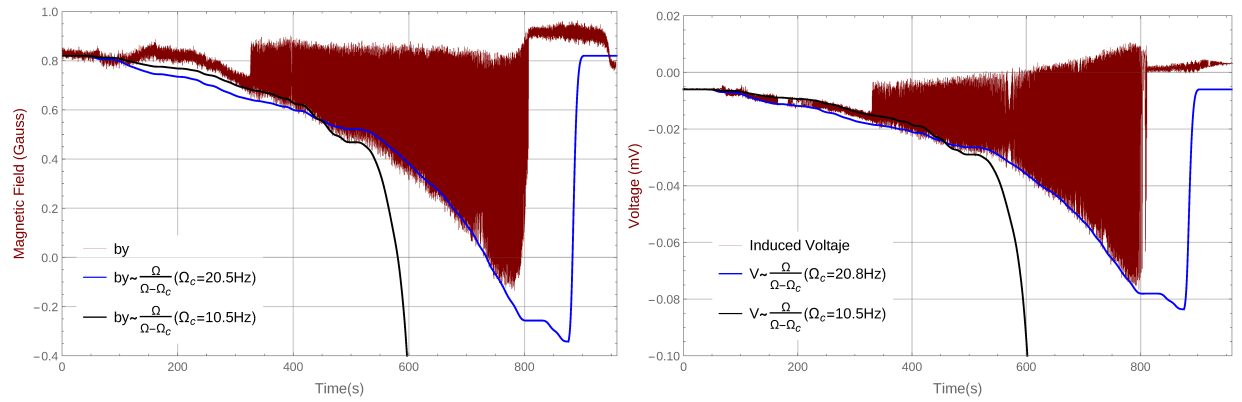

Figure 5: Comparison of the measured magnetic field and voltage with the expected variation $\sim \Omega /\left(\Omega_{c}-\Omega\right)$. The critical rotation rate which best fits the measurements is $\Omega_{c}=20.5 \mathrm{~Hz}\left(\Omega_{c}=20.8 \mathrm{~Hz}\right)$. The black curve corresponds to the expected theoretical variation function with $\Omega_{c}=10.5 \mathrm{~Hz}$.

4. Conclusions We report the first test results of the homopolar disc dynamo device proposed by Priede et al. 5 and constructed at CICATA-Querétaro in Mexico. Induced magnetic field and the voltage drop on the coil were measured for rotation rates up to $14 \mathrm{~Hz}$, at which the liquid metal started to leak from the outer sliding contact. Although the rotation rate was significantly above the theoretically predicted dynamo threshold of $10.4 \mathrm{~Hz}$, self-excitation of the magnetic 
field was not achieved. Instead of the steady magnetic field predicted by the theory we detected a strongly fluctuating magnetic field with a strength comparable to that of Earth's magnetic field which was accompanied by similar voltage fluctuations in the coil. These fluctuations seem to be caused by the intermittent electrical contact through the liquid metal. The experimental results suggest that the dynamo with the actual electrical resistance of liquid metal contacts could be excited at the rotation rate of around $21 \mathrm{~Hz}$ provided that the leakage of liquid metal is prevented.

Acknowledgments This work is supported by the National Council for Science and Technology of Mexico (CONACYT) through the project CB-168850 and by the National Polytechnic Institute of Mexico (IPN) through the project SIP-20171098. The first author thanks to M. Valencia, A. Pérez and G. García for their technical support during the experimental run.

\section{REFERENCES}

1. H. K. Moffat. Magnetic field generation in electrically conducting fluids. (Cambrige, UK, 1978).

2. R. Beck, A. Brandenburg, D. Moss and A. Shukurov Galactic magnetism: recent developments and perspectives. Annu. Rev. Astron. Astrophys., vol. 34 (1996), pp. 155-206.

3. E.-C. Bullard. The stability of a homopolar dynamo. Phil. Soc., vol. 51 (1955), pp. $744-760$.

4. K. H. Raedler, And M. Rheinhardt. Can a disc dynamo work in the laboratory?. Magnetohydrodynamics, vol. 38 (2002), pp. 211-217.

5. J. Priede And R. A. Avalos-Zúñiga. Feasible homopolar dynamo with sliding liquid-metal contacts. Physics letters A, vol. 377 (2013), pp. 2093-2096.

6. D. Maribo, M. Gavrilash, P. J. Reilly, W. A. Lynch, N. A. Sondergaard. Comparison of Several Liquid Metal Sliding Electric Contacts. Proceedings of the 56th IEEE Holm Conference on Electrical Contacts., (2010), pp. 1-7.

7. D. Maribo, And N. A. SondergaArd. Further studies of a low-melting point alloy used in a liquid metal current collector. IEEE Transactions on Components, Hybrids, and Manufacturing Technology, vol. 10 (1987), pp. 452-455.

8. C. E. Bello-Morales. Caracterización electromagnética del dínamo de disco con contactos de metal líquido. Master Thesis. CICATA-Qro./IPN, Mexico, 2016. 